\title{
Sentido y significado en las ocupaciones de las mujeres: una experiencia desde el municipio de Inzá, Cauca ${ }^{1}$
}

\section{Sense and Meaning in Occupations of Women: Experience from the Municipality of Inza Cauca}

\author{
Jeannette Amanda Méndez Montaño², Diana Carolina Bravo Castro ${ }^{3}$ \\ Naydu Yafith Camelo Valencia ${ }^{4}$, Derly Constanza Zúñiga Chaguala ${ }^{5}$
}

Recibido: Abril 62013 • Enviado para modificación: Mayo 222013 • Aceptado: Junio 32013

Méndez, J.; Bravo, D.; Camelo, N.; Zúñiga, D. (2013). Sentido y significado en las ocupaciones de las mujeres: Una experiencia desde el municipio de Inzá, Cauca. Revista Ocupación Humana 13 (1), pp. 19-30.

\begin{abstract}
Resumen: Las transformaciones asumidas por la mujer, le han permitido trascender en distintas ocupaciones y suplir sus necesidades a partir del contexto histórico-cultural en el que participa. El objetivo de la investigación fue identificar el Sentido y el Significado que tienen las ocupaciones para las mujeres del municipio de Inzá, Cauca. Para ello se usó la metodología de Investigación Acción Participativa como alternativa que favorece la construcción colectiva de conocimiento. El proceso permitió encontrar conclusiones alrededor de la dinámica necesaria entre los determinantes y los componentes planteados. A partir de esto se dan recomendaciones encaminadas al conocimiento de diversos usos del tiempo libre. Se rescata la participación como ocupación importante para las mujeres, ya que genera sentido de pertenencia y autovaloración, convirtiéndose en un escenario que favorece la re-significación de la vida.
\end{abstract}

Palabras clave: Mujer, Participación, Terapia Ocupacional, Género, Ocupación.

\begin{abstract}
Transformations assumed by women have allowed them to transcend in different occupations and meet their needs within the cultural historical context in which they participate. The objective of the research was to identify the sense and meaning that occupation has for these women in the municipality of Inzá, Cauca. The Participatory Action Research methodology was selected as an alternative that favors collective construction of knowledge. Conclusions were obtained through the process around the required dynamics among the established determinants and components and recommendations toward a different use of leisure time were done based on them. Participation is well recognized by women due to the sense of belonging and self-esteem that it generates giving a new meaning to their lives and becoming a scenario for personal development.
\end{abstract}

Key words: Women, Participation, Occupational Therapy, Gender, Occupation.

\footnotetext{
${ }^{1}$ Artículo basado en la investigación Construcciones de sentido y significado de las ocupaciones de las mujeres inzaeñas: aportes desde Terapia Ocupacional; trabajo ganador del Premio de investigación Alicia Trujillo Rojas, versión 2013, en la categoría Estudiantes.

2 Terapeuta Ocupacional, Magister en desarrollo educativo y social. Docente de planta, Departamento de la Ocupación Humana. Universidad Nacional de Colombia. jamendezm@unal.edu.co

${ }^{3}$ Terapeuta Ocupacional. Centro de Rehabilitación Integral Infantil Especializada RIIE. tocarolinabravo@gmail. com

${ }^{4}$ Terapeuta Ocupacional. Unidad de Salud Mental, Hospital La Victoria.nycamelov@unal.edu.co

${ }^{5}$ Terapeuta Ocupacional. Clínica del Country. to.derlyzuch@gmail.com
} 


\section{Introducción}

Las mujeres han asumido un papel cultural particular, a través de actividades remitidas a distinciones de género, atribuidas a partir de imaginarios construidos socioculturalmente, como la ejecución de labores domésticas, el cuidado del hogar, de los hijos y del esposo.

El sistema político del Cauca, excluyente y restrictivo, ha generado que la mujer deba ganar por sus méritos espacios de participación en la comunidad, superando la situación de violencia imperante en Colombia. Aunque la comunidad reconoce el ejercicio de participación de las mujeres, hay mayor valoración hacia actividades tradicionalmente asignadas a ellas: lavar, cocinar, recoger café, enseñar, cuidar hijos, animales y la huerta casera.

La participación, como ocupación, genera en la mujer sentido de pertenencia y autovaloración; debe recuperarse como escenario de desarrollo personal y re significación de la vida, porque favorece su empoderamiento transformando el sentido de su ser, sus actividades diarias, las formas de relacionarse con su comunidad y los significados sociales, enriqueciendo su Ser y proyectándose en su Llegar a Ser.

La escasa evidencia en Terapia Ocupacional sobre la relación entre Ser, Hacer, Llegar a Ser, Sentido, Significado y Perspectiva de Género, motivan la investigación con comunidades, promoviendo su participación activa en el proceso y enriqueciendo el conocimiento teórico.
El trabajo en el municipio de Inzá, Cauca, se vio enriquecido por las características del contexto sociopolítico y cultural propio de la región, al ser, junto al municipio de Páez, la región de Tierradentro, declarada por la UNESCO como Patrimonio Histórico de la Humanidad, por su diversidad sociocultural.

En la región se evidencia la fuerte influencia que ejerce el aspecto sociopolítico al enfrentarse la comunidad indígena con la campesina, en este contexto las mujeres desempeñan diferentes roles, organizándose a nivel político, productivo y reproductivo. La principal actividad económica es la agropecuaria y se caracteriza por producir el café más suave y de mejor calidad del país.

Este estudio se desarrolló partiendo de la hipótesis de la interacción que existe entre las determinantes ${ }^{6}$ de Ser, Hacer y Llegar a Ser, cuyas premisas han sido planteadas por Wilcock (1999); Significado, abordado por Trombly (1995) y Sentido, analizado por Irizar (2008); junto con los componentes, témporo espacial, personal, social, cultural y el contexto ambiental. Se tiene en cuenta también la perspectiva de género, que envuelve las actividades de las mujeres Inzaeñas.

Wilcock (1993, citado por Argüelles y Pérez 2008) plantea que la ocupación es el mecanismo mediante el cual las personas satisfacen sus necesidades básicas vinculadas con la supervivencia, la salud y la habilidad para prosperar en ambientes que les permiten crecer hasta alcanzar su

\footnotetext{
${ }^{6}$ Para esta investigación, las determinantes hacen referencia a los aspectos y perspectivas de la ocupación humana desde los cuales se partió para llevar a cabo todo el proceso.
} 
potencial. Esto es influenciado por fuerzas y valores socioculturales, a partir de lo cual, propone que la ocupación es una síntesis entre el Hacer, el Ser y el Llegar a Ser.

En cuanto al Hacer, Wilcock (1999), señala que la evolución humana ha estado colmada de haceres sucesivos y progresivos, los cuales permiten al ser humano sobrevivir, además de estimular, entender y excitar, a algunas personas, mientras que aburren, estresan, alienan o deprimen a otras, de acuerdo a lo que fue realizado. Esto permite llegar a un estado de satisfacción al Hacer, proporcionando el mecanismo para la interacción, el desarrollo y el crecimiento social, que constituye la pertenencia a una comunidad y la construcción de una identidad local.

Cuando se hace referencia a nuestra naturaleza, nuestra esencia y lo que nos distingue de los otros y que al mismo tiempo los atrae hacia nosotros como parte de las relaciones y de lo que hacemos, se habla del Ser. Este se asocia con la capacidad de elegir, de ser retado, de hallar el balance y con las oportunidades. También se asocia con los roles ocupacionales que manifiestan la esencia de cada persona y se consolida en el bienestar que experimentan las personas cuando existe el interés y ponen en práctica las habilidades para trabajar de forma eficaz y colaboradora con la comunidad.

El Llegar a Ser es el sentido del futuro del Ser, depende de lo que las personas hacen y son en el presente de su historia cultural. Involucra la noción del poder de facilitación que tiene la ocupación para el desarrollo, crecimiento y potencial de transformación y autorrealización humana que están presentes a lo largo de toda la vida. Se relaciona con la autonomía, constituyendo un agente de auto-creación y de expresión de los valores sociales, por ello cada persona estará siempre en un proceso continuo y constante de Llegar a Ser. En la investigación, este aspecto, fue analizado solamente desde la proyección y expectativas a futuro.

En lo que se refiere al Significado de la ocupación, de acuerdo con Trombly (1995), cuenta el valor de cumplimiento, logro o realización que una persona tiene de una meta. Emana del desempeño de actividades que son vistas como importantes socialmente, basándose en los valores que surgen de las experiencias personales y familiares; se deriva de la creencia entre la conexión mente-cuerpo durante el ciclo vital de la persona. Las ocupaciones que las personas realizan, ostentan un significado según el contexto y la cultura en la cual se encuentran inmersas.

En definitiva, los individuos pueden realizar la misma actividad, pero esta tendrá un significado diferente de acuerdo a los factores del contexto social, cultural, y político en el cual se encuentren inmersos.

En cuanto al Sentido de la ocupación, Irizar, (2008) denomina sentido a todo aquello capaz de impregnar significado, a todos los actos, relaciones y cosas que configuran la existencia personal. Es el "valor" personal asignado a las actividades y construido desde las experiencias sensoriales, afectivas, motoras, cognitivas, sociales y espirituales, que se acumulan a lo largo del ciclo vital. Agrega, que los sentidos construidos por el ser humano en su devenir histórico adquieren forma a 
partir de su relación con el paisaje, el ambiente, la lengua, la cultura, el amplio espectro de emociones posibles, al encontrar una forma ocupacional, entre otros aspectos acordes con su identidad, su historia y su pertenencia.

El valor personal depende de las experiencias vividas en la colectividad, sus deseos, sus valores, lo que quiere y genera una intención.

A continuación se definen los componentes que interactúan con las determinantes propuestas:

- Tiempo y espacio. Zemke (2004 citado por Méndez, 2011) propone el abordaje de estas nociones a partir de las experiencias subjetivas individuales, por lo que habla de temporalidad, haciendo referencia a la experiencia de cada persona con el tiempo, a medida que es moldeado por el desarrollo de ocupaciones; y a la espacialidad ocupacional, relacionada con nuestro reconocimiento, percepción y significado de lugar durante las acciones ocupacionales.

- Cultura. Krefting \& Krefting (1991 citado por McGruder, 2005) la refieren como la experiencia aprendida y compartida que proporciona al individuo y al grupo mecanismos eficaces para interactuar con los otros y con su entorno. Al hablar de cultura, se deben tener en cuenta sus atributos intrínsecos: es real, pues aunque no es tangible, sus efectos nos rodean; es aprendida, ya que aprendemos una forma de visualizar el mundo y de vivir en el mismo a través de las enseñanzas de nuestras familias, comunidades y medios de comunicación; es compartida, dado que se observa a través de las interacciones sociales; es maleable y dinámica, está cambiando constan- temente como consecuencia de las fuerzas, políticas, históricas, sociales y económicas.

- Individual. Este componente está relacionado con las capacidades físicas, mentales, espirituales, expectativas personales y lo que hace que cada ser humano sea único e irrepetible dentro de espacios socioculturales y ambientales específicos. Cuando se habla de lo individual se debe hablar también de lo colectivo ya que hay una construcción subjetiva del individuo en relación a los otros (Virno, 2003 citado por Rubio y Sanabria 2010).

- Social. De acuerdo con Trujillo (2002), este componente tiene que ver con la capacidad de la persona para establecer relaciones interindividuales en diferentes escenarios, participar en actividades de intercambio social amplio y contribuir con causas de beneficio social; Spencer (2005), menciona que el contexto social se relaciona con la localidad y variedad de redes de apoyo personal que los seres humanos buscan y establecen.

- Contexto-ambiente. Desde la perspectiva del contexto, se asume el contexto temporal (edad cronológica, estado de desarrollo, ciclo de vida y estado de salud) por los significados sociales y culturales que se tienen durante el ciclo vital y el contexto medioambiental (dimensiones físicas, sociales y culturales) que influye en las opciones y acciones de las personas. (Dunn, Brown \& Youngstrom 2003, citados por Méndez 2011). La relación entre la persona y el contexto es dinámica en tanto las experiencias de la persona generan cambios en su actuación, modificándola según sus necesidades, habilidades y destrezas, y 
los nuevos significados que la persona otorga a su ocupación.

- Género. El término surgió con los movimientos feministas de la década del 70 y se refiere a una construcción cultural, social e histórica basada en la diferencia biológica de los sexos que dio origen a una valoración social de lo femenino y de lo masculino (Jara R., 2004, citado por Heredia, 2004). Aunque el concepto fue reducido al aspecto biológico, actualmente, retoma aspectos sociales, culturales y lo que respecta a las creencias, dando cuenta de su transformación y re significación a través del tiempo. La perspectiva de género es el estudio de la manera como se construyen, analizan y viven en la cultura y la historia los distintos roles genéricos, comprendiendo el análisis de todas las relaciones inter genéricas e intra genéricas. Uno de sus fines es posibilitar la construcción subjetiva y social de una configuración de las dimensiones humanas a partir de la re significación de la historia, la sociedad, la cultura y la política desde y con las mujeres. Así, el género se convierte en una categoría de análisis que recorre todas las dimensiones y niveles de una sociedad (García, Vélez \& Vivas 2004).

En ese sentido, conviene resaltar la relación que guarda esta perspectiva con el concepto de empoderamiento, adquiriendo diferentes significados de acuerdo al contexto social y político en que se defina. Se deriva de la base según la cual, las personas en situación de subordinación, cuentan con mayor fuerza para participar y defender sus derechos cuando se unen con otras personas en pro del alcance de objetivos en común; dicho agrupamiento, puede facilitar la toma de conciencia sobre estas situaciones, favoreciendo el camino hacia el cambio.

En el caso de las mujeres, incluye el cambio individual y colectivo, así como la transformación de procesos y estructuras que reproducen situaciones desfavorecidas y subordinadas, promoviendo la adquisición de recursos, conciencia y habilidades para su vencimiento, aumentando su poder sobre recursos y decisiones que afectan su vida e incrementado su influencia y participación en el cambio social, con el fin de formar parte de la sociedad en real igualdad de condiciones.

\section{Metodología}

Se utiliza la metodología de Investigación Acción Participativa (I.A.P.) la cual permite conocer las dinámicas sociales, culturales y económicas de la comunidad, y el papel que cumplen las mujeres Inzaeñas dentro de estas.

El tipo de estudio descriptivo, permite observar la ocupación a partir de los aspectos de Ser, Hacer, Llegar a Ser, Sentido y Significado que las mujeres le asignan a sus ocupaciones.

En este trabajo de investigación participaron tres grupos de mujeres, cada uno de ellos tuvo un proceso de selección diferente debido a las características del contexto, contando con un total de 60 mujeres agrupadas de la siguiente manera:

- Mujeres vinculadas a la Asociación Campesina de Inzá Tierradentro (A.C.I.T.). Tienen una formación política, están vinculadas y participan en actividades desarrolladas por el comité de mujeres de la A.C.I.T., participaron 16 lideresas. 
- Mujeres no vinculadas a la A.C.I.T. Habitan en la cabecera municipal de Inzá, no se encuentran vinculadas al comité por no tener el tiempo necesario para participar o no están de acuerdo con sus ideales; participaron 13 mujeres.

- Mujeres de la vereda de Guanacas. Madres de los niños y niñas en condiciones de vulnerabilidad que estudian en la Institución Educativa Promoción Social Guanacas (I.E.P.S.), participaron 31 mujeres.

Inicialmente se identificaron las necesidades de las mujeres inzaeñas, las de las mujeres de la A.C.I.T., docentes y campesinos de la A.C.I.T., con quienes se llevó a cabo la realización de entrevistas semi-estructuradas, encuentros conversacionales y visitas domiciliarias de acuerdo a los contextos de los tres grupos poblacionales de mujeres. Estas estrategias permitieron conocer más a fondo las actividades en las que se desempeñan las mujeres en su cotidianidad, de acuerdo a su contexto histórico-cultural. Una vez identificadas, fueron descritas para su posterior análisis, teniendo en cuenta aquellas que, de acuerdo a lo manifestado por las mujeres y a la experiencia vivencial del día a día, representan mayor interés.

Durante el proceso, la comunidad hizo parte activa de la investigación, emprendiendo acciones junto al equipo investigativo, dirigidas hacia la comprensión crítica y a la transformación de su realidad, basándose en la experiencia y el conocimiento tradicional. De esta manera se fortalecieron las dinámicas sociales, favoreciendo la búsqueda de estrategias para generar cambios en cuanto a los aspectos que pudiesen limitar sus dinámicas organizativas. El proceso se llevó a cabo de la siguiente manera:

En la I.E.P.S. Guanacas, el acercamiento a las mujeres de la comunidad se realizó mediante visitas domiciliarias a 31 familias, habitantes de veredas aledañas a Guanacas. La población se definió a partir de la caracterización realizada por el coordinador y docentes de primaria, referente a las familias de estudiantes en condiciones de vulnerabilidad (violencia intrafamiliar, bajo rendimiento académico, dificultades de comportamiento, hacinamiento, ausencia temporal de los padres por actividades laborales desempeñadas en otras ciudades y por ello, designación del cuidado de los niñas y niños a otros familiares, hijos mayores o vecinos). A la vez se acordó, realizar observaciones del ambiente domiciliario y brindar indicaciones terapéuticas orientadas a la necesidad e importancia de mejorar algunas condiciones del ambiente físico y familiar en aras de favorecer el proceso escolar de los menores ${ }^{7}$. Las visitas se distribuyeron por equipos conformados por un docente, un estudiante de grado $10^{\circ}$ y una Terapeuta Ocupacional en formación. Se realizó la entrevista a la mujer a cargo del hogar, en la mayoría de los casos fue la madre de los estudiantes, en otros casos, las cuidadoras de los niños y niñas, y en una mínima proporción al padre.

\footnotetext{
${ }^{7}$ Los estudiantes que participaron en la investigación lo hicieron motivados por los docentes, con el fin de evidenciar parte del proceso metodológico de la misma, además de contextualizarse sobre las necesidades de su comunidad. En este sentido, los docentes se interesaron por conocer el contexto en el que sus estudiantes se desenvuelven partiendo del punto de vista de Terapia Ocupacional.
} 
El acercamiento con el Comité de Mujeres de la A.C.I.T., se realizó a través de encuentros conversacionales, que permitieron construir la entrevista semiestructurada, con el propósito de contribuir al proceso de fortalecimiento de la organización y observar sus desarrollos, luego de un encuentro que se llevó a cabo en la cabecera municipal de Inzá en el año 2008. Se identificaron mujeres lideresas con fuertes procesos de empoderamiento dentro de su comunidad, representantes de cada vereda aledaña al municipio, y otras que formaban parte de la directiva del comité, en total 16 mujeres.

Para el caso de las mujeres no vinculadas a la A.C.I.T., se realizó la entrevista semiestructurada a 13 mujeres, lideresas en las actividades que realizan diariamente: administración de uno o más negocios (restaurantes, venta de alimentos sin procesar y suministros para las diferentes labores del campo, crianza y venta de especies menores como pollos); trabajo con la comunidad en la realización de actividades para el bien común; docencia en instituciones educativas y otros oficios como el de belleza corporal. Estas mujeres son: madres solteras cabeza de familia, trabajadoras independientes y profesoras que, con empeño, fortaleza y perseverancia, han alcanzado logros importantes en sus vidas, reflejados en la dedicación que demuestran al desempeñarse en sus actividades cotidianas.

\section{Resultados}

Los resultados se presentan desde el Ser, el Hacer, el Llegar a Ser; y el Sentido y Significado que las mujeres les asignan a sus ocupaciones.

\section{Ser: concepciones de ser mujer}

Las mujeres, definen ser mujer, como la esencia de ser persona, la base del hogar, lo principal, el eje, el motor de la familia, de la sociedad y de una nación. La mayoría, manifiesta que ser mujer es sentirse bien y realizadas con lo que hacen, sentirse a gusto con los trabajos que realizan a diario; hacerse respetar y respetarse, valorarse a sí mismas y a las demás. Asimismo, definen el ser mujer desde sentimientos, virtudes y valores como la sensibilidad, solidaridad, amabilidad, comprensión, amor y compañerismo. Ser mujer, para ellas, es generar vida, dar frutos, concebir los hijos y darles amor y ternura así como tener un hogar, encaminando sus representaciones hacia el vínculo que establecen con la creación de Dios, en tanto que es una bendición dar y formar la vida. Consideran que la mujer es un ser que Dios creó en la tierra para que ayude al hombre, sin sentirse inferior a él, lo cual les genera orgullo, permitiéndoles exaltar su identidad personal y reconocer su esencia; tienen en cuenta sus características físicas y sus propios valores resaltando sus fortalezas y debilidades.

Las mujeres manifiestan que la mujer y el hombre se complementan; sin embargo, han vivido experiencias tristes y manifiestan su deseo de no haber sido mujeres, pues este hecho representa una labor ardua, de mucha responsabilidad. Tienen obligaciones con el hogar y la familia y trabajan muy duro, pues en muchas ocasiones, los compañeros sentimentales las abandonan con sus hijos, lo cual deja ver las dificultades en cuanto a la va 
loración de sí mismas y de su hacer cotidiano. Algunas mujeres definen el ser mujer, desde el desempeño de sus roles, en tanto les da identidad y un lugar en la sociedad, permitiéndoles obtener reconocimiento de su hacer cotidiano como personas y no como mujeres.

\section{Hacer}

Las mujeres inzaeñas participantes, consideran importante realizar actividades de la vida diaria, particularmente el aseo personal, porque les permite una adecuada presentación personal, lo que consideran fundamental para el establecimiento de relaciones sociales y políticas y con ello construir vínculos que enriquecen el desempeño de su rol como mujeres empoderadas. Sin embargo, algunas mujeres dejan de lado su propio cuidado (presentación personal y acicalamiento) y su bienestar, y se dedican solo a actividades que les han sido asignadas socialmente, como el trabajo material y el cuidado de sus hijos. Asimismo, resaltan la importancia de realizar actividades domésticas como: lavar, cocinar, cuidar la huerta que tienen en casa, alistar a los hijos para ir al colegio, estar pendientes de ellos al regresar y acompañarles en el desarrollo de las tareas escolares.

Igualmente es relevante mantener el hogar organizado y limpio, ya que representa un espacio en el cual comparten saberes y experiencias con sus hijos; aunque, sienten que la responsabilidad y obligación de realizarlas recae solamente sobre ellas. Esta situación se incrementa por ser cabeza de hogar o por tener una pareja disfuncional que no participe de forma equitativa en las actividades propias del hogar, teniendo que delegar funciones del cuidado de los hijos y arreglo del hogar a los hijos mayores. Poco participan en actividades distintas a las del cuidado del hogar por considerar que este es más importante para lograr el bienestar de los miembros de su familia, esperando que sus hijos correspondan satisfactoriamente con las actividades escolares y un buen comportamiento, como gesto de recompensa y agradecimiento hacia ellas. No obstante, algunas ven la realización de estas actividades como una obligación.

En cuanto a las actividades que realizan en su tiempo libre, la mayoría referencia las mismas del cuidado del hogar y de sus hijos, sin contar con tiempo para desempeñar actividades distintas a estas; sin embargo, otras mujeres reconocen la importancia del descanso realizando actividades que van de la mano con sus gustos e intereses. No se evidencia iniciativa para que ellas mismas emprendan acciones encaminadas hacia su propio bienestar y mejoramiento de su calidad de vida. Por lo anterior, el uso del tiempo libre no es asumido como un espacio de esparcimiento, ocio, desarrollo personal y exploración de gustos e intereses, sino que es tomado para la realización de actividades productivas.

En las mujeres inzaeñas que participan con/en la comunidad, se evidencia que esto es importante, ya que a través de esta, comparten experiencias y saberes en torno a las actividades que constituyen su hacer, conocen y ayudan a su comunidad, toman decisiones, establecen y fortalecen relaciones interpersonales, logros que no encuentran en sus hogares o en la 
ejecución del trabajo material. El sentido otorgado a la participación social como una ocupación, está mediado por la gratificación personal que resulta de las actividades que realizan, no se sienten obligadas a hacerlas y representan un espacio en el cual la valoración de su hacer es más tangible. La participación incrementa el liderazgo y empoderamiento, y los resultados de sus acciones permiten la gestión de proyectos, la solución de las diferentes dificultades que hacen parte de su cotidianidad, así como el desarrollo y cumplimiento de objetivos, metas y propósitos para el bien común y personal.

\section{Llegar a ser}

Las proyecciones de las mujeres inzaeñas, reflejan su deseo por desempeñar actividades relacionadas con sus gustos, intereses, expectativas y sueños a futuro como: descansar, comer bien, pasear, compartir con su familia y con la comunidad, mantener la casa organizada, trabajar, estudiar, bailar, escuchar música, realizar actividades manuales, vestirse bien y arreglarse para que sus hijos y esposos se sientan bien, lo cual las hace sentir bien consigo mismas; el hecho de regresar a sus ciudades de origen, aunque algunas refieren que no cambian la vida del campo por la de la ciudad, "...cambiar de ambiente", tener una vida mejor y disfrutar de lo que se ha conseguido y aprendido; incluso consideran importante, darse una "segunda oportunidad con alguien". Algunas mujeres manifiestan que no han pensado en nada para un futuro; no obstante, les interesa el trabajo político y electoral, así como la organización de las mujeres para conocer otras experiencias y empoderarse.

De la misma manera, consideran importante y desean que sus hijos continúen sus estudios de bachillerato y universitarios, que logren sus propósitos y mejoren su calidad de vida. En un futuro, quieren descansar junto a ellos, con el fin de que ellos no repitan sus experiencias, dejando ver su interés por salir adelante, aunque en muchas de ellas, se evidencia la falta de iniciativa para la puesta en marcha de este propósito. Entre sus expectativas y proyectos, se encuentra el realizar mejoras físicas a sus viviendas, dado que algunos espacios de las mismas se encuentran en condiciones poco favorables. Asimismo, desean tener un negocio, vivienda o finca propia en la que puedan tener animales y contar con una huerta, con el fin de vivir y trabajar con mayor tranquilidad junto a sus familias y alcanzar mayor estabilidad económica y emocional. Algunas mujeres que cuentan con una finca quieren tecnificarla, tener herramientas para mejorar el trabajo material y de este modo, vivir más tranquilas, ampliar la extensión de tierra con la que cuentan, trabajar y sostener a sus familias. El servicio a la comunidad hace parte de sus expectativas lo cual expresan en su deseo por compartir con otras personas lo que han aprendido y ayudar a niños que no tienen familia, además de servir a Dios mediante sus acciones.

Una gran parte de las mujeres considera importante dar continuidad y culminar sus estudios ya sean de bachillerato o universitarios (derecho, ingeniería agronomía, psicología, enfermería y pedagogía), o realizar cursos a nivel técnico o tecnológico 
de manicure, sistemas, modistería o enfermería. Reconocen que para lograrlo es necesario salir del pueblo y estudiar en la ciudad, aun sabiendo que particularmente, poseen menores oportunidades para capacitarse o especializarse respecto a los hombres, debido en parte, al tiempo que le dedican al cuidado de sus hijos y su hogar. Muchas otras, logran vincularse a procesos de formación educativa o laboral, porque cuentan con el apoyo de su esposo o familiares, o cuentan con el recurso económico para tal fin. Las mujeres consideran que las personas de su familia valoran lo que ellas hacen, por el reconocimiento verbal, simbólico y material (celebración de fechas especiales), esperando que sus hijos reconozcan lo que ellas hacen y les retribuyan con su comportamiento y cumplimiento de las actividades escolares y la colaboración en la realización de algunas actividades del cuidado del hogar.

\section{Discusión y conclusiones}

Los resultados permiten evidenciar que el sentido que las mujeres participantes otorgan a sus ocupaciones está impregnado en gran medida, por los significados sociales, es decir, el sentido se estructura en función de los significados y el hacer, los cuales están enriquecidos por componentes culturales, sociales y el contexto ambiental, encontrando diferencias en la dinámica de las mujeres que participan en actividades de índole político y aquellas que participan en actividades de carácter comunitario.

El proceso investigativo da cuenta de la premisa de Wilcock (1999), acerca de la ocupación como meca- nismo para satisfacer las necesidades básicas, vinculadas con la habilidad para prosperar en ambientes que permiten el crecimiento personal, tendiente al alcance del propio potencial. Al mismo tiempo es influenciada por fuerzas y valores socioculturales, condensándose en el Hacer, el Ser y el Llegar a Ser, viéndose reflejado en las perspectivas que surgen de las mujeres inzaeñas en cuanto al valor y la importancia que tiene la ejecución de las distintas actividades cotidianas dentro de su vida rutina diaria.

De la misma manera, permite confirmar el planteamiento de Trombly (1995), respecto al significado de la ocupación, ya que este emana del desempeño de distintas actividades vistas como importantes socialmente, evidenciando la manera en que el contexto y la cultura de las mujeres inzaeñas interactúan y adquieren importancia, a la vez que dan paso a la construcción de sentido y significado de las ocupaciones.

En ese orden de ideas, es evidente, que el sentido que otorgan las mujeres a sus ocupaciones, se constituye a partir de su relación con todos los elementos de su entorno, lo que en la presente investigación se ha denominado componentes: (social, individual, contexto-ambiental, cultural y témporo espacial), apoyando la afirmación de Irizar (2008), frente a la construcción que realiza el ser humano sobre los sentidos, la cual está influenciada por su devenir histórico, su relación con el paisaje, el ambiente, la lengua, la cultura, así como el amplio espectro de emociones posibles al encontrar una forma ocupacional.

Es claro que las mujeres continúan ocupándose en trabajos vistos tradi- 
cionalmente como femeninos, de menor retribución y valoración social que aquellos desempeñados por los hombres, dejando ver que aún existe una fuerte presencia del sistema patriarcal.

De otro modo, las mujeres inzaeñas carecen de espacios, tiempo e iniciativa para el desarrollo de actividades que hagan parte de su tiempo libre. Esto genera una sobrecarga de actividades, haciendo necesaria la re significación del mismo, de manera que permita construir nuevos sentidos y significados de vida alrededor del aprovechamiento del tiempo libre en la construcción de su proyecto vital. Se observa la necesidad que tienen algunas de ellas para expresar sus experiencias, ideas, pensamientos, opiniones y sentimientos a las personas de la comunidad; ser escuchadas y tenidas en cuenta para actividades diferentes a las del campo y el hogar, reconociéndose como seres, y ante todo, como mujeres valiosas con infinitas habilidades y capacidades, visibilizándose más allá del aspecto físico.

Se destacaron aspectos limitantes durante el proceso: la fuerte influencia ejercida por el contexto socio-político de la región, sumado a los factores histórico-culturales y a los ideales político-organizacionales, que hacen susceptible el desarrollo y son capaces de sesgar el proceso de investigación, obstaculizando los alcances de la misma. Afectan la cobertura de atención en otros sectores vulnerables de la población abordada, que presentan iguales o mayores necesidades de las identificadas al inicio conjuntamente con la comunidad; además, la falta de recursos económicos y de talento humano, como constitución de redes de apoyo político y social, también limitan la ejecución de las acciones orientadas a la promoción y prevención, y por ende, la satisfacción de las expectativas de otras comunidades e incluso, de otros grupos poblacionales involucrados, por lo que se hace necesario establecer alianzas con entidades nacionales gubernamentales y no gubernamentales interesadas en el aporte al bienestar y el desarrollo particular de la comunidad.

Cabe resaltar la disposición, interés, motivación, colaboración y compromiso de las personas que participaron activamente dentro del proceso, que generó en la comunidad el deseo de darle continuidad en los lugares donde se inició, así como la extensión a otras veredas, lo cual se reflejó notablemente en la comunidad de la escuela de Guanacas, donde fue posible evidenciar la transformación y el impacto generados en la mejora de su calidad de vida, a través del seguimiento asertivo y oportuno de indicaciones terapéuticas brindadas.

A partir de lo mencionado, es posible deducir que las mujeres logran su empoderamiento a través del proceso de organización y participación política que desarrollan en su devenir temporal y espacial, transformando constantemente el sentido de ser mujer, de sus actividades cotidianas, sus expectativas, la forma como pueden relacionarse con su comunidad $y$, de sus significados, viéndose reflejado en el enriquecimiento de su Ser y la proyección de su Llegar a Ser, de acuerdo a las necesidades, expectativas, metas y objetivos enmarcados en sus estilos de vida ancestrales, sociales, culturales y políticos que han imperado tradicionalmente. 
En conclusión, es de relevancia social dar continuidad a investigaciones que aborden la relación e interacción que existe entre el Ser, el Hacer, el Llegar a Ser, el Sentido y el Significado de las ocupaciones, y la forma en que los componentes individual, social, temporal, espacial, cultural y contexto ambiental se superponen implícitamente en las mismas, destacando así, el valor y la importancia que tienen las ocupaciones desempeñadas por las mujeres colombianas, y perseguir la ampliación continua de los horizontes investigativos en torno a la Ocupación Humana.

\section{Referencias}

Argüelles A. \& Pérez B. (2008) Sentido y lugar de la ocupación en la práctica profesional: "análisis en cuatro campos de acción". Universidad Nacional de Colombia, Departamento de la Ocupación Humana, Bogotá, Colombia.

Bonaccorsi, N. (2004). Los debates feministas sobre la división sexual de los espacios público y privado. En Heredia N., Videla M. (Ed.), pensamiento feminista II, aportes para un nuevo andamiaje social, mujer, género y feminismo (89-118). Argentina: Ediciones C.E.N.

Bravo C., Camelo N. \& Zúñiga C., (2011) Construcciones de sentido y significado de las ocupaciones de las mujeres inzaeñas: aportes desde terapia ocupacional. Universidad Nacional de Colombia, Departamento de la Ocupación Humana, Bogotá, Colombia.

Dunn, Brown \& Youngstrom (2003) Ecological Model of Occupation. En Kramer, Hinojosa.

Brasic (Ed.), Perspectives in Human Occupation. USA: Lippincontt Williams \& Wilkins.

García D., Vélez O. \& Vivas M. (2004). Reflexiones en torno al feminismo y al gé- nero; Teología y género (175-183). Bogotá: Digiprint Editores.

Irizar L. (2008). Tras las huellas del sentido. Bogotá: Corporación Pensamiento Siglo XXI Konrad Adenauer Universidad Sergio Arboleda.

Jara R. (2004); Mujer, género, feminismo. En Heredia N., Videla M. (Eds.) pensamiento feminista II, aportes para un nuevo andamiaje social, mujer, género y feminismo (7-9). Argentina: Ediciones C.E.N.

Krefting L. \& Krefting D. (1991). Cultural influences on performance. En C. Christiansen y C. Baum (Eds). Occupational therapy: Overcoming human performance déficits (101-124). Thorofare, NJ:Slack

Larson E., Wood W., \& Clark F. (2005). Ciencia ocupacional: desarrollo de la ciencia y la práctica de la ocupación a través de una disciplina académica. EnCrepeau, Cohn \& Schell (10a․ Ed.), Willard y Spackman, Terapia Ocupacional (15-24). España: Editorial médica panamericana.

Spencer J. (2005). Evaluación contextual del desempeño. En Crepeau, Cohn \& Schell (10a․ Ed.), Willard y Spackman, Terapia Ocupacional (427-444). España: Editorial médica panamericana.TN Relaciones (s.f.) En Mujer: empoderamiento de las mujeres. Recuperado de http://www. tnrelaciones.com/empoderamiento/

Trombly C. (1995). Occupation: purposefulness and meaningfulness as therapeutic mechanisms. American Journal of Occupational Therapy, 49(10), pp. 960-971.

Trujillo A. (2002). Terapia Ocupacional, conocimiento y práctica en Colombia. Bogotá: Editorial Universidad Nacional de Colombia.

Virno P. (2003). Gramática de la multitud. Madrid: Editorial Traficantes de Sueños.

Wilcock A. (1999). Reflections on doing, being and becoming. Australian Occupational Therapy 46(1), pp. 1-11.

Zemke R. (2004). The 2004 Eleanor Clark Slagle Lecture. Time, space, and the kaleidoscopes of occupation. American Journal of Occupational Therapy. 58(6), pp. 608-620. 